\title{
Pliocene-Pleistocene dispersal bring along low inter species diversity between Vimba species based on multilocus analysis
}

\author{
Gökhan Kalayc1 ${ }^{1}$ \\ 1 Recep Tayyip Erdoğan University, Faculty of Fisheries, Department of Basic Sciences, 53100 Rize, Turkey \\ http://zoobank.org/A65F15B9-00BD-4F26-BA1D-4B1EEC4AB30F \\ Corresponding author: Gökhan Kalaycı (gokhan.kalayci@erdogan.edu.tr)
}

Academic editor: Nicolas Hubert • Received 23 October 2021 Accepted 15 February 2022 • Published 25 February 2022

\begin{abstract}
This study investigates phylogenetic and phylogeographic relationships of Vimba species using mitochondrial cytochrome b (cyt b) (1023 bp) and cytochrome c oxidase subunit I (COI) barcoding region (652 bp) genes. Ninety-one samples from 36 populations for the cyt b gene and 67 samples from 20 populations for the COI were analyzed. We identified 29 haplotypes and calculated overall haplotype diversity as Hd: $0.907 \pm 0.015$ for cyt $b$. We also identified $13 \mathrm{COI}$ haplotypes and calculated overall haplotype diversity as $0.826 \pm 0.026$ for this marker. The phylogenetic analysis of Vimba species reveals the presence of four clades, based on concatenated cyt $b$ and COI sequences. The first and second clade consist of Vimba vimba Western lineage, and Vimba vimba Caspian lineage, while the third and fourth clade consist of Vimba mirabilis and Vimba melanops. Based on haplotype network analyses and phylogeographic inferences, the Vimba genus is monophyletic, and its species dispersed in the Pleistocene era.
\end{abstract}

\section{Key Words}

cyt b, genetic diversity, Phylogeography, Vimba bream, Vimba mirabilis

\section{Introduction}

As a member of the Leuciscidae family, the genus Vimba is distributed throughout almost all Eurasia and consists of three species: Vimba vimba, Vimba melanops, and Vimba mirabilis. V. vimba was initially described as Cyprinus vimba L. from several Swedish lakes in Scandinavia, the North Sea, coastal waters of Baltic Sea basins, and, subsequently, after the description, it was also found in the Caspian, Black Sea, Marmara Sea basins, and the Rhine River. In Anatolia, $V$. vimba is distributed from the Marmara basin up to Büyük Menderes, Eğirdir Lake, Köprüçay and Eşen rivers in the south, and Kizilırmak in the east. $V$. melanops was described initially from the Meriç (Evros) River and its distribution now extends within the borders of Turkey, Greece, Bulgaria, and Macedonia in the North Aegean basin from Meriç to the Pinios River. The Anatolian endemic $V$. mirabilis was detected only in the type locality Büyük Menderes and two individuals in Bafa Lake in Southwest Anatolia (Bogutskaya 1997). According to Crivelli (1996),
$V$. mirabilis is under threat of extinction due to the water intake from the basin for drinking and irrigation.

Since then, a few taxonomic developments of the genus Vimba have occurred, like the subalpine Vimba lineage previously identified as Vimba elongata, but now considered a synonym of Vimba vimba. Acanthobrama mirabilis was synonymized with Vimba vimba tenella by Durand et al. (2002), as A. mirabilis belongs to the Vimba clade. But, Perea et al. (2010) found Acanthobrama mirabilis is the synonym of $V$. mirabilis based on genetic evidence. Also, in some literature, $V$. vimba, distributed in the Caspian Sea and Caspian bream, has been identified as Vimba persa (Hänfling et al. 2009; Naseka and Bogutskaya 2009; Chaichi et al. 2011a, 2011b; Mohamadian et al. 2012).

Globally, Vimba genus has been well studied in terms of growth parameters and its parasites, but genetic studies are more limited (Zardoya and Doadrio (1999), Durand et al. (2002), Hänfling et al. (2009), Perea et al. (2010), Triantafyllidis et al. (2011), Geiger et al. (2014), Schönhuth et al. (2018). In particular, Hänfling et al. (2009) discovered 
the phylogeographic origin and colonization factor of V. vimba, but other studies have quite limited samples.

Phylogeographic and phylogenetic studies have been made on some European freshwater fishes such as $\mathrm{Al}$ burnoides, Barbus, Capoeta, Gobio, Squalius, Telestes and typically uncovered distinct patterns according to mitochondrial cytochrome b (cyt b) and cytochrome c oxidase I (COI) genes. In order to study systematics and phylogeny of Leuciscidae and its congeners, cyt $b$ and COI sequence analysis have been demonstrated to be useful DNA markers. (Zardoya and Doadrio 1999; Durand et al. 2000, 2002; Tsigenopoulos et al. 2002; Levin et al. 2012; Bektas et al. 2017; Turan et al. 2018; Aksu and Bektaş 2019).

In European rivers, freshwater fishes have largely colonized the Black Sea upstream, up rivers such as the Dnieper and the Danube. Freshwater fishes contain numerous lineages with genetic divergences representing separation over the past $2 \mathrm{Myr}$ in this region. Several of these have clear geographic distributions and provide evidence of older Black Sea-Caspian Sea divergence. Interglacial and postglacial expansions also indicate colonization of Western Europe from numerous major refugia, particularly the Black Sea, Dnieper-Volga, Danube, Rhine- Rhone, Elbe, and other rivers. The influence of older water bodies such as the Ponto-Caspian Sea and recent great periglacial lakes and floods is also apparent (Hewitt 2004).

While it frequently highlighted that the Pleistocene increased speciation rates, molecular data have recently revealed that species diverged in the Pleistocene and Pliocene in Europe (Hewitt 2000).

In the current study, mtDNA sequences were used (cyt b, COI barcoding) to examine the biogeography of Vimba populations, applying a phylogeographic approach. The objective is to reveal the phylogenetic relationships and genetic diversity of Vimba species whose populations are currently decreasing.

\section{Materials and methods}

\section{Sample collection, DNA extraction, and sequencing}

All currently recognized taxa of Vimba were included in our dataset. We sequenced 68 Vimba specimens collected from their distribution ranges in Turkey and further included sequences of 23 specimens from NCBI GenBank. Vimba species of Turkey were collected from 14 sampling sites of drainages of the Black and Aegean and Marmara seas, comprising type localities or type basins (Fig. 1, Table 1). The animals were experimented with as per the Republic of Turkey animal welfare laws, guidelines, and policies, and was approved by the Republic of Turkey Recep Tayyip Erdogan University Local Ethics Committee for Animal Experiments (Decision No: 2014/72). For faunal surveys, fishes were collected, surgical procedures were performed only for excision of fin clips after anesthesia with MS222, and fin clips were further preserved in $70 \%$ ethanol for genetic studies. In our experiments, none of the fishes were distressed by the experimental conditions. Specimens and tissue vouchers from Turkey were kept in DNA Collection and Zoology Museum, Faculty of Fisheries, Recep Tayyip Erdoğan University, Rize. Total DNA was extracted from fin clips via Qiacube automated DNA/RNA purification system by using Qiagen DNeasy Blood \& Tissue Kits (Qiagen, Hilden, Germany). Both the quality and quantity of DNA were checked on a NanoDrop 2000/c spectrophotometer (Thermo Scientific, Rockford, IL, USA) and 0.8\% agarose gel electrophoresis. Cyt b (1023 bp) gene was amplified by the newly designed primer set B-cytbF (5'-GAAGAACCACCGTTGTWVTTCAAC-3') and the B-cytbR (5'- CGGATTACAAGACCGATGC -3'), and COI barcoding gene (652 bp) was amplified by the FishF1 (5'-TCAACCAACCACAAAGACATTGGCAC-3') and FishR1 (5'-TAGACTTCTGGGTGGCCAAAGAATCA-3') (Ward et al. 2005). PCR reactions were performed in a $50 \mu \mathrm{L}$ reaction volume containing $5 \mu \mathrm{L} 10 \mathrm{x}$ PCR buffer, $100 \mathrm{ng}$ template DNA, $0.5 \mathrm{mM}$ dNTPs mix, $3 \mathrm{mM} \mathrm{MgCl} 2,0.5 \mathrm{mM}$ of each primer, and $1 \mu \mathrm{L}$ Taq DNA polymerase (New England Biolabs). The polymerization was carried out under the following conditions: initial denaturation at $95{ }^{\circ} \mathrm{C}$ for $30 \mathrm{~s}$, denaturation at $95^{\circ} \mathrm{C}$ for $30 \mathrm{~s}$, annealing at $55^{\circ} \mathrm{C}$ for $50 \mathrm{~s}$ for cyt b and $58^{\circ} \mathrm{C}$ for $45 \mathrm{~s}$ for COI, extension at $68^{\circ} \mathrm{C}$ for $1 \mathrm{~min}$ through 35 cycles, and a final extension at $68^{\circ} \mathrm{C}$ for 5 min using Biorad T100 (Bio-Rad, Hercules, CA, USA) thermal cycler. The PCR products were run and visualized under UV Quantum-Capt ST4 system (Vilber Lourmat, France) also, purified and sequenced at Macrogen Europa Inc. (Amsterdam, Netherlands) (Turan et al. 2018).

\section{Genetic structure and phylogenetic analysis}

Clustal W (Thompson et al. 1994) algorithm was implemented in Bioedit v7.2.5 (Hall 1999) software to align cyt $\mathrm{b}$ and COI barcoding regions. Haplotype number $(\mathrm{H})$, polymorphic and variable sites, haplotype diversity $(\mathrm{Hd})$, and nucleotide diversity $(\pi)$ were computed for each species by DnaSP version 6.12.03 (Rozas et al. 2017) program. Nucleotide frequencies and transition/transversion rate were calculated by MEGA X (Kumar et al. 2018). Molecular Variance (AMOVA) was analyzed using the Arlequin v3.5.1.2 (Excoffier and Lischer 2010) software to calculate genetic variation among and within the groups. The haplotypes were submitted to the NCBI GenBank with accession numbers OK493404-OK493416 for cyt b OK444819-OK444823 for COI barcoding region. Cyt $\mathrm{b}$ and COI sequences were concatenated $(1675 \mathrm{bp})$ for further phylogenetic inferences. The $\mathrm{TrN}+\mathrm{G}$ model:- $\ln =2905.1890$ was the best-fit nucleotide substitution model for the concatenated dataset according 
Table 1. List of sequences analyzed in this study with information on drainage, GenBank no, haplotype no, and country of origin.

\begin{tabular}{|c|c|c|c|c|c|c|c|c|c|c|}
\hline \multirow{2}{*}{$\begin{array}{l}\text { Locality } \\
\text { no }\end{array}$} & \multirow[t]{2}{*}{ Species } & \multirow[t]{2}{*}{ Locality } & \multirow[t]{2}{*}{ Coordinate } & \multicolumn{3}{|c|}{ cyt b } & \multicolumn{3}{|c|}{$\mathrm{COI}$} & \multirow[t]{2}{*}{ Reference } \\
\hline & & & & $\mathbf{N}$ & Genbank no & $\begin{array}{c}\text { Haplotype } \\
\text { no }\end{array}$ & $\mathbf{N}$ & Genbank no & $\begin{array}{c}\text { Haplotype } \\
\text { no }\end{array}$ & \\
\hline 1 & Vimba vimba & $\begin{array}{c}\text { Kırmir stream, Ankara, } \\
\text { Turkey }\end{array}$ & $\begin{array}{l}40^{\circ} 14^{\prime} 10.5^{\prime \prime} \mathrm{N} \\
32^{\circ} 15^{\prime} 41.2^{\prime \prime} \mathrm{E}\end{array}$ & 7 & OK493404 & $\mathrm{H} 1$ & 3 & OK444821 & $\mathrm{H} 7$ & This study \\
\hline 2 & Vimba vimba & $\begin{array}{c}\text { Binkılıç stream, Black sea, } \\
\text { Istanbul, Turkey }\end{array}$ & $\begin{array}{l}41^{\circ} 22^{\prime} 48.3^{\prime \prime N} \\
28^{\circ} 17^{\prime} 46.0^{\prime \prime} \mathrm{E}\end{array}$ & 9 & $\begin{array}{l}\text { OK493404, } \\
\text { OK493407, } \\
\text { OK493408 }\end{array}$ & $\mathrm{H} 1, \mathrm{H} 4, \mathrm{H} 5$ & 8 & OK444820 & $\mathrm{H} 6$ & This study \\
\hline 3 & Vimba vimba & $\begin{array}{c}\text { Koca stream, Balıkesir, } \\
\text { Turkey }\end{array}$ & $\begin{array}{l}39^{\circ} 46^{\prime} 55.2^{\prime \prime N} \\
27^{\circ} 35^{\prime} 46.2^{\prime \prime} \mathrm{E}\end{array}$ & 4 & $\begin{array}{l}\text { OK493404, } \\
\text { OK493406, } \\
\text { OK493409 }\end{array}$ & $\mathrm{H} 1, \mathrm{H} 3, \mathrm{H} 6$ & 4 & $\begin{array}{l}\text { OK444820, } \\
\text { OK444821 }\end{array}$ & $\mathrm{H} 6, \mathrm{H} 7$ & This study \\
\hline 4 & Vimba vimba & $\begin{array}{l}\text { Koca çayı stream, } \\
\text { Çanakkale, Turkey }\end{array}$ & $\begin{array}{l}39^{\circ} 48^{\prime} 52.9^{\prime \prime} \mathrm{N} \\
27^{\circ} 13^{\prime} 46.1^{\prime \prime} \mathrm{E}\end{array}$ & 3 & $\begin{array}{l}\text { OK493405, } \\
\text { OK493406 }\end{array}$ & $\mathrm{H} 2, \mathrm{H} 3$ & 3 & $\begin{array}{l}\text { OK444820, } \\
\text { OK444821 }\end{array}$ & $\mathrm{H} 6, \mathrm{H} 7$ & This study \\
\hline 5 & Vimba vimba & $\begin{array}{c}\text { Aydınlar stream, Zonguldak, } \\
\text { Turkey }\end{array}$ & $\begin{array}{l}41^{\circ} 13^{\prime} 47.1^{\prime \prime N} \\
31^{\circ} 27^{\prime} 11.2^{\prime \prime} \mathrm{E}\end{array}$ & 2 & OK493410 & $\mathrm{H7}$ & & & & This study \\
\hline 6 & Vimba vimba & Aksu stream, Düzce, Turkey & $\begin{array}{l}40^{\circ} 45^{\prime} 49.0^{\prime \prime} \mathrm{N} \\
30^{\circ} 57^{\prime} 43.0^{\prime \prime} \mathrm{E}\end{array}$ & 3 & OK493411 & $\mathrm{H} 8$ & & & & This study \\
\hline 7 & Vimba vimba & $\begin{array}{l}\text { Büyük Melen stream, } \\
\text { Düzce, Turkey }\end{array}$ & $\begin{array}{l}40^{\circ} 50^{\prime} 08.0^{\prime \prime} \mathrm{N} \\
31^{\circ} 06^{\prime} 35.0^{\prime \prime} \mathrm{E}\end{array}$ & 4 & OK493411 & $\mathrm{H} 8$ & & & & This study \\
\hline 8 & Vimba vimba & $\begin{array}{c}\text { Yenice stream, Zonguldak, } \\
\text { Turkey }\end{array}$ & $\begin{array}{l}41^{\circ} 20^{\prime} 27.6^{\prime \prime N} \\
32^{\circ} 04^{\prime} 40.8^{\prime \prime} \mathrm{E}\end{array}$ & 5 & OK493410 & $\mathrm{H} 7$ & & & & This study \\
\hline 9 & Vimba vimba & $\begin{array}{c}\text { Çayagzı stream, Düzce, } \\
\text { Turkey }\end{array}$ & $\begin{array}{l}41^{\circ} 05^{\prime} 27.2^{\prime \prime} \mathrm{N} \\
31^{\circ} 13^{\prime} 18.5^{\prime \prime} \mathrm{E}\end{array}$ & 3 & $\begin{array}{l}\text { OK493410, } \\
\text { OK493411 }\end{array}$ & $\mathrm{H} 7, \mathrm{HB}$ & & & & This study \\
\hline 10 & Vimba vimba & Iznik lake, Kocaeli, Turkey & $\begin{array}{l}40^{\circ} 26^{\prime} 18.1^{\prime \prime N} \\
29^{\circ} 38^{\prime} 03.5^{\prime \prime} \mathrm{E}\end{array}$ & 10 & OK493406 & $\mathrm{H} 3$ & & & & This study \\
\hline 11 & Vimba vimba & $\begin{array}{l}\text { Suat Ugurlu Dam lake, } \\
\text { Samsun, Turkey }\end{array}$ & $\begin{array}{l}41^{\circ} 01^{\prime} 52.4 " \mathrm{~N} \\
36^{\circ} 38^{\prime} 33.4^{\prime \prime} \mathrm{E}\end{array}$ & 2 & $\begin{array}{l}\text { OK493412, } \\
\text { OK493413 }\end{array}$ & $\mathrm{H} 9, \mathrm{H} 10$ & & & & This study \\
\hline 12 & Vimba vimba & $\begin{array}{c}\text { Curonian Lagoon, Baltic } \\
\text { Sea basin, Lithuania }\end{array}$ & $\begin{array}{l}55^{\circ} 42^{\prime} 18.0^{\prime \prime} \mathrm{N} \\
20^{\circ} 00^{\prime} 00.0^{\prime \prime} \mathrm{E}\end{array}$ & 1 & GQ279763 & $\mathrm{H} 11$ & & & & $\begin{array}{l}\text { Hänfling et al. } \\
2009\end{array}$ \\
\hline 13 & Vimba vimba & $\begin{array}{c}\text { Danube I, Black Sea basin } \\
\text { Germany }\end{array}$ & $\begin{array}{l}48^{\circ} 53^{\prime} 24.0^{\prime \prime N} \\
11^{\circ} 48^{\prime} 54.0^{\prime \prime} \mathrm{E}\end{array}$ & 1 & GQ279762 & $\mathrm{H} 12$ & & & & $\begin{array}{l}\text { Hänfling et al. } \\
2009\end{array}$ \\
\hline 14 & Vimba vimba & $\begin{array}{c}\text { Elbe, North Sea basin, } \\
\text { Germany }\end{array}$ & $\begin{array}{l}51^{\circ} 28^{\prime} 30.0 " \mathrm{~N} \\
11^{\circ} 588^{\prime} 01.2^{\prime \prime} \mathrm{E}\end{array}$ & 1 & GQ279761 & $\mathrm{H} 13$ & & & & $\begin{array}{l}\text { Hänfling et al. } \\
2009\end{array}$ \\
\hline 15 & Vimba vimba & $\begin{array}{c}\text { Olandsan, Baltic Sea basin, } \\
\text { Sweden }\end{array}$ & $\begin{array}{l}60^{\circ} 20^{\prime} 24.0^{\prime \prime} \mathrm{N} \\
17^{\circ} 31^{\prime} 19.2^{\mathrm{E}} \mathrm{E}\end{array}$ & 1 & GQ279756 & $\mathrm{H} 14$ & & & & $\begin{array}{l}\text { Hänfling et al. } \\
2009\end{array}$ \\
\hline 16 & Vimba vimba & $\begin{array}{l}\text { Mondsee, Danube, Black } \\
\text { Sea basin, Germany }\end{array}$ & $\begin{array}{l}47^{\circ} 49^{\prime} 40.8^{\prime \prime} \mathrm{N} \\
13^{\circ} 23^{\prime} 02.4^{\prime \prime} \mathrm{E}\end{array}$ & 2 & $\begin{array}{l}\text { GQ279755, } \\
\text { AY026405 }\end{array}$ & $\mathrm{H} 18$ & & & & $\begin{array}{l}\text { Hänfling et al. } \\
2009 \text { Durand et } \\
\text { al. } 2002\end{array}$ \\
\hline 17 & Vimba vimba & $\begin{array}{l}\text { Eder, Weser, North Sea } \\
\text { basin, Germany }\end{array}$ & $\begin{array}{l}51^{\circ} 09^{\prime} 18.0^{\prime \prime} \mathrm{N} \\
8^{\circ} 54^{\prime} 07.2^{\prime \prime} \mathrm{E}\end{array}$ & 1 & GQ279755 & $\mathrm{H} 18$ & & & & $\begin{array}{l}\text { Hänfling et al. } \\
2009\end{array}$ \\
\hline 18 & Vimba vimba & $\begin{array}{c}\text { Tuzlov, Don, Sea of Azov } \\
\text { basin, Russia }\end{array}$ & $\begin{array}{l}49^{\circ} 58^{\prime} 58.8^{\prime \prime N} \\
42^{\circ} 01^{\prime} 04.8^{\prime \prime} \mathrm{E}\end{array}$ & 1 & GQ279751 & $\mathrm{H} 15$ & & & & $\begin{array}{l}\text { Hänfling et al. } \\
2009\end{array}$ \\
\hline 19 & Vimba vimba & $\begin{array}{l}\text { Seversky, Donetz Don, Sea } \\
\text { of Azov basin, Russia }\end{array}$ & $\begin{array}{l}47^{\circ} 37^{\prime} 37.2 " \mathrm{~N} \\
40^{\circ} 53^{\prime} 16.8^{\prime \prime} \mathrm{E}\end{array}$ & 1 & GQ279751 & $\mathrm{H} 15$ & & & & $\begin{array}{l}\text { Hänfling et al. } \\
2009\end{array}$ \\
\hline 20 & Vimba vimba & $\begin{array}{c}\text { Samur, Caspian Sea basin } \\
\text { Russia }\end{array}$ & $\begin{array}{l}41^{\circ} 52^{\prime} 26.8^{\prime \prime N} \\
48^{\circ} 33^{\prime} 34.9^{\prime \prime} \mathrm{E}\end{array}$ & 1 & GQ279765 & $\mathrm{H} 16$ & & & & $\begin{array}{l}\text { Hänfling et al. } \\
2009\end{array}$ \\
\hline 21 & Vimba vimba & $\begin{array}{c}\text { Sea of Azov, Sea of Azov } \\
\text { basin Ukraine }\end{array}$ & $\begin{array}{l}46^{\circ} 03^{\prime} 50.4 " \mathrm{~N} \\
36^{\circ} 36^{\prime} 54.0^{\prime \prime} \mathrm{E}\end{array}$ & 2 & $\begin{array}{l}\text { GQ279754, } \\
\text { GQ279752 }\end{array}$ & $\mathrm{H} 1 \mathrm{H} 3$ & & & & $\begin{array}{l}\text { Hänfling et al. } \\
2009\end{array}$ \\
\hline 22 & Vimba vimba & $\begin{array}{c}\text { Kuban, Sea of Azov basin, } \\
\text { Russia }\end{array}$ & $\begin{array}{l}45^{\circ} 11^{\prime} 56.4 " \mathrm{~N} \\
37^{\circ} 42^{\prime} 54.0^{\prime \prime} \mathrm{E}\end{array}$ & 1 & GQ279753 & $\mathrm{H} 10$ & & & & $\begin{array}{l}\text { Hänfling et al. } \\
2009\end{array}$ \\
\hline 23 & Vimba vimba & $\begin{array}{l}\text { Tsymlyansk Reservoir, Don, } \\
\text { Sea of Azov basin, Russia }\end{array}$ & $\begin{array}{l}47^{\circ} 45^{\prime} 56.2^{\prime \prime} \mathrm{N} \\
42^{\circ} 49^{\prime} 18.8^{\prime \prime} \mathrm{E}\end{array}$ & 1 & GQ279751 & $\mathrm{H} 15$ & & & & $\begin{array}{l}\text { Hänfling et al. } \\
2009\end{array}$ \\
\hline 24 & Vimba vimba & $\begin{array}{l}\text { Dagomys, northeastern } \\
\text { Black Sea basin, Russia }\end{array}$ & $\begin{array}{l}43^{\circ} 40^{\prime} 01.2^{\prime \prime} \mathrm{N} \\
39^{\circ} 39^{\prime} 07.2^{\prime \prime} \mathrm{E}\end{array}$ & 1 & GQ279750 & $\mathrm{H} 3$ & & & & $\begin{array}{l}\text { Hänfling et al. } \\
2009\end{array}$ \\
\hline 25 & Vimba vimba & $\begin{array}{l}\text { Libechovka river, Elbe basin, } \\
\text { Czech Republic }\end{array}$ & $\begin{array}{l}50^{\circ} 28^{\prime} 45.0^{\prime \prime} \mathrm{N} \\
14^{\circ} 29^{\prime} 07.5^{\prime} \mathrm{E} \\
\text { (predict) }\end{array}$ & 1 & HM560237 & $\mathrm{H} 17$ & 1 & HM560383 & $\mathrm{H} 6$ & Perea et al. 2010 \\
\hline 26 & Vimba vimba & $\begin{array}{c}\text { Bashly-chai, Caspian Sea } \\
\text { Basin, Russia }\end{array}$ & $\begin{array}{l}42^{\circ} 20^{\prime} 37.0^{\prime \prime} \mathrm{N} \\
48^{\circ} 05^{\prime} 30.8^{\prime \prime} \mathrm{E}\end{array}$ & 1 & GQ279765 & $\mathrm{H} 16$ & & & & $\begin{array}{l}\text { Hänfling et al. } \\
2009\end{array}$ \\
\hline 27 & Vimba vimba & $\begin{array}{c}\text { Lake Sapanca, Sakarya, } \\
\text { Turkey }\end{array}$ & $\begin{array}{l}40^{\circ} 43^{\prime} 14 " \mathrm{~N} \\
30^{\circ} 17^{\prime} 41^{\prime \prime} \mathrm{E}\end{array}$ & & & & 20 & $\begin{array}{l}\text { KC501853- } \\
\text { KC501872 }\end{array}$ & $\mathrm{H} 13$ & $\begin{array}{c}\text { Keskin \& Atar } \\
2013\end{array}$ \\
\hline 28 & Vimba vimba & $\begin{array}{c}\text { Gönen drainage, Çanakkale, } \\
\text { Turkey }\end{array}$ & $\begin{array}{l}39^{\circ} 56^{\prime} 45.6 " \mathrm{~N} \\
27^{\circ} 20^{\prime} 13.2^{\prime \prime} \mathrm{E}\end{array}$ & & & & 2 & $\begin{array}{l}\text { KJ554799, } \\
\text { KJ554924 }\end{array}$ & $\mathrm{H} 11, \mathrm{H} 12$ & Geiger et al. 2014 \\
\hline 29 & Vimba vimba & Egirdir Lake, Isparta, Turkey & $\begin{array}{l}38^{\circ} 02^{\prime} 00.9^{\prime \prime} \mathrm{N} \\
30^{\circ} 52^{\prime} 24.3^{\prime \prime} \mathrm{E} \\
\text { (predict) }\end{array}$ & & & & 2 & $\begin{array}{l}\text { MW940905, } \\
\text { MW940906 }\end{array}$ & $\mathrm{H} 10, \mathrm{H} 6$ & Eren,H. (unp.) \\
\hline 30 & Vimba vimba & $\begin{array}{c}\text { Biga drainage, Bursa, } \\
\text { Turkey }\end{array}$ & $\begin{array}{l}40^{\circ} 12^{\prime} 18.0^{\prime \prime} \mathrm{N} \\
29^{\circ} 05^{\prime} 13.2^{\prime \prime} \mathrm{E}\end{array}$ & & & & 1 & KJ554609 & $\mathrm{H} 6$ & Geiger et al. 2014 \\
\hline 31 & Vimba vimba & $\begin{array}{c}\text { Koeprue drainage, Isparta, } \\
\text { Turkey }\end{array}$ & $\begin{array}{l}37^{\circ} 31^{\prime} 40.8^{\prime \prime} \mathrm{N} \\
31^{\circ} 16^{\prime} 08.4^{\prime \prime} \mathrm{E}\end{array}$ & & & & 2 & $\begin{array}{l}\text { KJ554606, } \\
\text { KJ554754 }\end{array}$ & $\mathrm{H} 6$ & Geiger et al. 2014 \\
\hline 32 & Vimba vimba & Danube R., Slovakia & $\begin{array}{l}48^{\circ} 04^{\prime} 04.2^{\prime \prime} \mathrm{N}, \\
17^{\circ} 09^{\prime} 53.2^{\prime \prime} \mathrm{E} \\
\text { (predict) }\end{array}$ & 1 & AY026404 & $\mathrm{H} 1$ & & & & $\begin{array}{l}\text { Durand et al. } \\
2002\end{array}$ \\
\hline 33 & $\begin{array}{l}\text { Vimba } \\
\text { melanops }\end{array}$ & $\begin{array}{c}\text { Inece stream, Kırklareli, } \\
\text { Turkey }\end{array}$ & $\begin{array}{l}41^{\circ} 41^{\prime} 34.0 " \mathrm{~N} \\
27^{\circ} 04^{\prime} 59.0^{\circ} \mathrm{E}\end{array}$ & 4 & $\begin{array}{l}\text { OK493415, } \\
\text { OK493416 }\end{array}$ & $\mathrm{H} 27 \mathrm{H} 28$ & 2 & OK444819 & $\mathrm{H} 1$ & This study \\
\hline 34 & $\begin{array}{l}\text { Vimba } \\
\text { melanops }\end{array}$ & $\begin{array}{l}\text { Evros, Aegean Sea basin, } \\
\text { Greece }\end{array}$ & $\begin{array}{l}40^{\circ} 50^{\prime} 42.0^{\prime \prime N} \\
26^{\circ} 01^{\prime} 22.8^{\prime \prime} \mathrm{E}\end{array}$ & 1 & GQ279757 & $\mathrm{H} 26$ & & & & $\begin{array}{l}\text { Hänfling et al. } \\
2009\end{array}$ \\
\hline
\end{tabular}




\begin{tabular}{|c|c|c|c|c|c|c|c|c|c|c|}
\hline \multirow{2}{*}{$\begin{array}{l}\text { Locality } \\
\text { no }\end{array}$} & \multirow[t]{2}{*}{ Species } & \multirow[t]{2}{*}{ Locality } & \multirow[t]{2}{*}{ Coordinate } & \multicolumn{3}{|c|}{ cyt b } & \multicolumn{3}{|c|}{$\mathrm{COI}$} & \multirow[t]{2}{*}{ Reference } \\
\hline & & & & $\mathbf{N}$ & Genbank no & $\begin{array}{l}\text { Haplotype } \\
\text { no }\end{array}$ & $\mathbf{N}$ & Genbank no & $\begin{array}{c}\text { Haplotype } \\
\text { no }\end{array}$ & \\
\hline 35 & $\begin{array}{l}\text { Vimba } \\
\text { melanops }\end{array}$ & Biserska River, Greece & $\begin{array}{l}40^{\circ} 55^{\prime} 08.5^{\prime \prime} \mathrm{N}, \\
26^{\circ} 11^{\prime} 48.0^{\prime \prime} \mathrm{E} \\
\text { (predict) }\end{array}$ & 1 & MG806725 & $\mathrm{H} 25$ & 1 & MG806910 & $\mathrm{H} 1$ & $\begin{array}{c}\text { Schonhuth et al. } \\
2018\end{array}$ \\
\hline 36 & $\begin{array}{l}\text { Vimba } \\
\text { melanops }\end{array}$ & River Strymon, Greece & $\begin{array}{l}41^{\circ} 43^{\prime} 53.5^{\prime \prime} \mathrm{N} \\
23^{\circ} 09^{\prime} 30.9^{\prime \prime} \mathrm{E} \\
\text { (predict) }\end{array}$ & 2 & $\begin{array}{l}\text { AF090778, } \\
\text { HM560236 }\end{array}$ & $\mathrm{H} 24 \mathrm{H} 21$ & 1 & HM560382 & $\mathrm{H} 4$ & $\begin{array}{c}\text { Zardoya \& } \\
\text { Doadrio, } 1999 \\
\text { Perea et al } 2010\end{array}$ \\
\hline 37 & $\begin{array}{l}\text { Vimba } \\
\text { melanops }\end{array}$ & $\begin{array}{l}\text { Pinios, Aegean Sea basin, } \\
\text { Greece }\end{array}$ & $\begin{array}{l}39^{\circ} 39^{\prime} 57.6^{\prime \prime} \mathrm{N} \\
22^{\circ} 14^{\prime} 02.4^{\prime \prime} \mathrm{E}\end{array}$ & 2 & $\begin{array}{l}\text { GQ279758, } \\
\text { GQ279759 }\end{array}$ & $\mathrm{H} 23 \mathrm{H} 22$ & & & & $\begin{array}{l}\text { Hänfling et al. } \\
2009\end{array}$ \\
\hline 38 & $\begin{array}{l}\text { Vimba } \\
\text { melanops }\end{array}$ & $\begin{array}{c}\text { Aliakmon R. Kaloneri, } \\
\text { Greece }\end{array}$ & $\begin{array}{l}40^{\circ} 17^{\prime} 26.5^{\prime \prime} \mathrm{N}, \\
21^{\circ} 28^{\prime} 17.9^{\prime \prime} \mathrm{E} \\
\text { (predict) }\end{array}$ & 1 & HM560235 & $\mathrm{H} 21$ & & & & Perea et al. 2010 \\
\hline 39 & $\begin{array}{l}\text { Vimba } \\
\text { melanops }\end{array}$ & Volvi lake, Greece & $\begin{array}{l}40^{\circ} 39^{\prime} 36.0^{\prime \prime} \mathrm{N}, \\
23^{\circ} 32^{\prime} 24.0^{\prime \prime} \mathrm{E}\end{array}$ & 1 & AY026403 & $\mathrm{H} 29$ & 3 & $\begin{array}{l}\text { HQ600801 } \\
\text { HQ600803 }\end{array}$ & $\mathrm{H} 1$ & $\begin{array}{c}\text { Triantafyllidis } \\
\text { et al. } 2011 \\
\text { Durand et al. } 2002\end{array}$ \\
\hline 40 & $\begin{array}{l}\text { Vimba } \\
\text { melanops }\end{array}$ & Kerkini lake, Greece & $\begin{array}{l}41^{\circ} 06^{\prime} 36.0^{\prime \prime} \mathrm{N} \\
23^{\circ} 03^{\prime} 00.0^{\prime \prime} \mathrm{E}\end{array}$ & & & & 3 & $\begin{array}{l}\text { HQ600804. } \\
\text { HQ600806 }\end{array}$ & $\begin{array}{c}\mathrm{H} 1, \mathrm{H} 3 \\
\mathrm{H} 4\end{array}$ & $\begin{array}{l}\text { Triantafyllidis et } \\
\text { al. } 2011\end{array}$ \\
\hline 41 & $\begin{array}{l}\text { Vimba } \\
\text { melanops }\end{array}$ & $\begin{array}{c}\text { Biserska R., Evros drainage, } \\
\text { Bulgaria }\end{array}$ & $\begin{array}{l}41^{\circ} 51^{\prime} 18.0^{\prime \prime} \mathrm{N} \\
25^{\circ} 55^{\prime} 22.8^{\prime \prime} \mathrm{E}\end{array}$ & & & & 3 & $\begin{array}{l}\text { KJ554935, } \\
\text { KJ554568, } \\
\text { KJ554722 }\end{array}$ & $\mathrm{H} 1$ & Geiger et al. 2014 \\
\hline 42 & $\begin{array}{l}\text { Vimba } \\
\text { melanops }\end{array}$ & $\begin{array}{c}\text { Charmanlijskaja drainage, } \\
\text { Bulgaria }\end{array}$ & $\begin{array}{l}41^{\circ} 53^{\prime} 20.4^{\prime \prime} \mathrm{N} \\
25^{\circ} 41^{\prime} 13.2^{\prime \prime} \mathrm{E}\end{array}$ & & & & 1 & KJ554876 & $\mathrm{H} 2$ & Geiger et al. 2014 \\
\hline 43 & $\begin{array}{l}\text { Vimba } \\
\text { melanops }\end{array}$ & Vardar drainage, Greece & $\begin{array}{l}40^{\circ} 59^{\prime} 16.8^{\prime \prime} \mathrm{N} \\
22^{\circ} 33^{\prime} 28.8^{\prime \prime} \mathrm{E}\end{array}$ & & & & 2 & $\begin{array}{l}\text { KJ554926, } \\
\text { KJ554576 }\end{array}$ & $\mathrm{H} 5$ & Geiger et al. 2014 \\
\hline 44 & $\begin{array}{l}\text { Vimba } \\
\text { mirabilis }\end{array}$ & $\begin{array}{l}\text { Akçay stream, Büyük } \\
\text { Menderes, Aydın, Turkey }\end{array}$ & $\begin{array}{l}37^{\circ} 45^{\prime} 34.0^{\prime \prime} \mathrm{N} \\
28^{\circ} 20^{\prime} 07.0^{\prime \prime} \mathrm{E}\end{array}$ & 9 & $\begin{array}{l}\text { OK493414, } \\
\text { AY026410 }\end{array}$ & $\mathrm{H} 20 \mathrm{H} 19$ & 4 & $\begin{array}{l}\text { OK444822, } \\
\text { OK444823 }\end{array}$ & $\mathrm{H} 8, \mathrm{H} 9$ & $\begin{array}{l}\text { This study, Durand } \\
\text { et al. } 2002\end{array}$ \\
\hline 45 & $\begin{array}{l}\text { Vimba } \\
\text { mirabilis }\end{array}$ & $\begin{array}{l}\text { Çine stream, Büyük } \\
\text { Menderes, Aydın, Turkey }\end{array}$ & $\begin{array}{l}37^{\circ} 45^{\prime} 43.8^{\prime \prime} \mathrm{N} \\
27^{\circ} 50^{\prime} 13.1^{\prime \prime} \mathrm{E}\end{array}$ & 4 & OK493414 & $\mathrm{H} 2 \mathrm{O}$ & 1 & KJ554739 & $\mathrm{H} 8$ & $\begin{array}{c}\text { This study, Geiger } \\
\text { et al. } 2014\end{array}$ \\
\hline
\end{tabular}

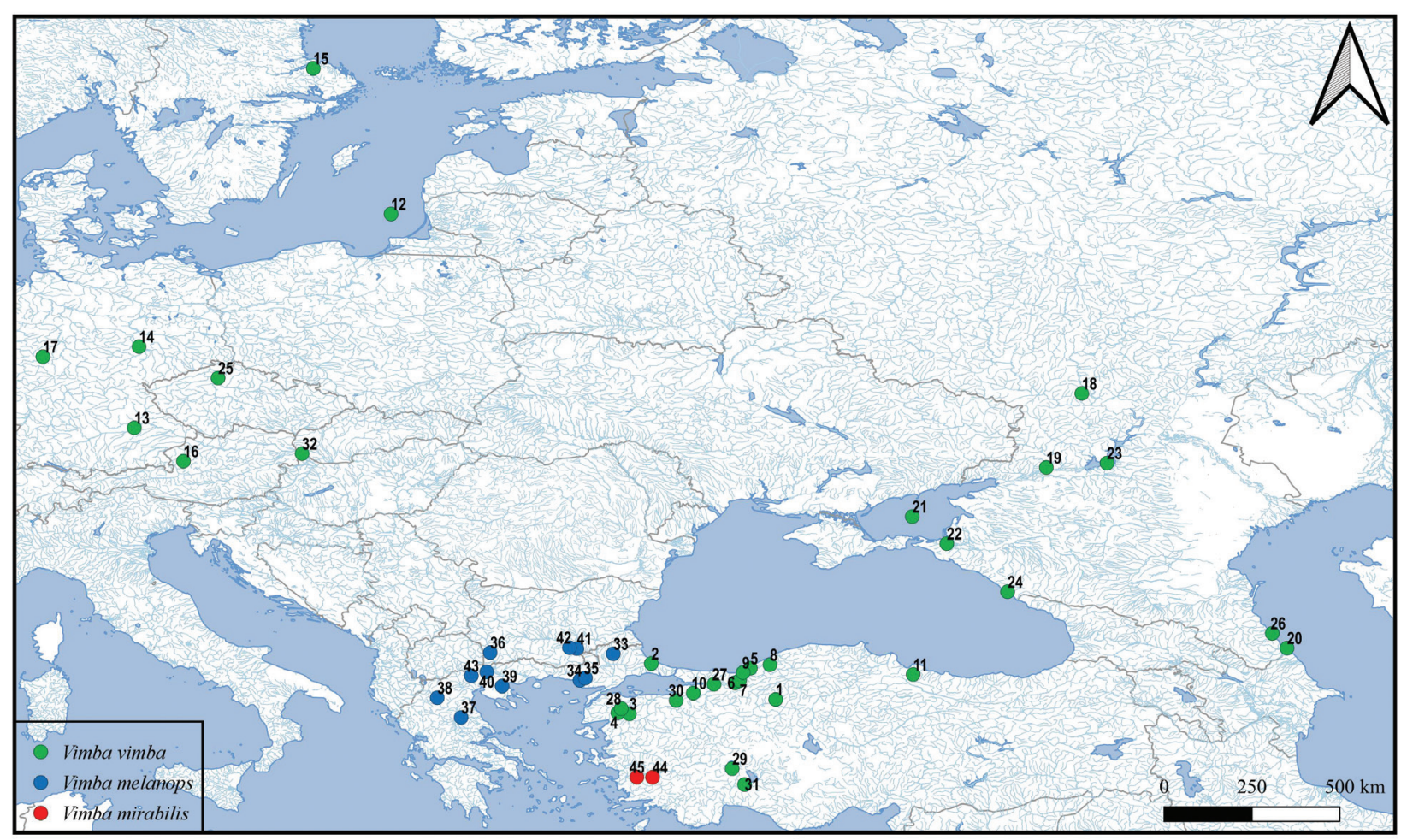

Figure 1. Map showing the analyzed population stations.

to Akaike information criterion (AIC) and Bayesian information criterion (BIC), as implemented in jModeltest v. 0.0.1 (Posada, 2008). Phylogenetic relationships among haplotypes and species were estimated by the maximum likelihood (ML) method using PhyML (Guindon et al. 2010) with 1000 bootstrap. Similarly, the Bayesian inference (BI) analysis was run in the MrBayes 3.1.2 software (Ronquist and Huelsenbeck 2003), using the Metropolis-coupled Markov chain
Monte Carlo (MCMC) algorithm from randomly generated starting trees for five million generations with sampling taking place in every 1000 generations. The initial $25 \%$ of saved trees sampled in each MCMC run were discarded as burn-in. In all phylogenetic analyses, Blicca bjoerkna (AP009304) was selected as outgroup taxa. Haplotypes' network inference was constructed through a median-joining (MJ) algorithm (Bandelt et al. 1999) implemented in Network 5.0.0.1 software 
(www.fluxus-engineering.com). MEGA X (Kumar et al. 2018) software was used to calculate pairwise genetic distance among the species using the Kimura 2-parameter substitution model (Kimura 1980).

We estimated divergence times using StarBEAST (Ogilvie et al. 2017), which was implemented in BEAST 2.6.0 (Bouckaert et al. 2014). Compared with standard BEAST, StarBEAST better accounts for species trees vs. gene trees and intraspecific vs. interspecific events. Species were delimited according to the individual grouping recovered by previous phylogenetic analyses. Thus, the analysis was conducted with 5 groups of individuals consisting of $V$. vimba (Caspian), $V$. vimba (Western), V. mirabilis, V. melanops, and outgroup Blicca bjoerkna. The molecular clock calibration was based on the divergence rate of cyt $b$ gene in Leuciscinae of $0.4 \%$ per lineage per million years, as determined by Perea et al. (2010), and this rate was used by Buj et al. (2019) and Viñuela Rodríguez et al. (2020). Based on a Yule speciation prior and a strict clock model, branch rate estimates were calculated. Partitioned cyt $b$ and COI dataset were used for the molecular clock analysis and the partitions were linked except for substitution models. The substitution models were used the TrN+G:-ln = 1878.5334 (Tamura and Nei 1993) and the HKY:-ln = 1063.2973 (Hasegawa et al. 1985) for cyt $b$ and COI barcoding region, respectively, with Gamma site heterogeneity for both markers. The number of MCMC steps (chain length) was ten million, with parameters logged every 1000 generations. The residual parameters were default parameters of the software. Tree results were summarized in TreeAnnotator v.2.6.0 software with 10\% burn-in to get a maximum clade credibility tree. Node bars, height median with height 95\% HPD, and node labels were mapped on the tree with FigTree v1.4.4 (Rambaut 2018). Geological scale was plotted using the geoscalePhylo function in R package strap (Bell and Lloyd 2015). Effective sample size (ESS) and convergence of parameters was estimated using Tracer v.1.6 (Rambaut and Drummond 2013). The effective sample sizes for all parameters of interest were greater than 200 .

\section{Results}

\section{Genetic diversity and species divergence}

The nucleotide sequences of the cyt $b$ gene region (1023 bp) were studied in 91 specimens from three species of Vimba (Additional Table 1). The average nucleotide frequencies were estimated as $26.9 \% \mathrm{~A}, 29.7 \% \mathrm{~T}, 28.0 \% \mathrm{C}$, and $15.4 \% \mathrm{G}$. The transition/transversion rate $\mathrm{k} 1=115.99$ (purines), $\mathrm{k} 2=34.563$ (pyrimidines) and the overall transition/transversion bias were estimated as $\mathrm{R}=31.458$. The haplotype number was identified as 29: 18 from $V$. vimba $(\mathrm{N}=66)$, nine from $V$. melanops $(\mathrm{N}=12)$, and two from $V$. mirabilis $(\mathrm{N}=13)$ (Additional Table 1). We identified 65 variable sites, of which 36 were parsimony-informative. Species-specific nucleotide (SSN) positions in the mitochondrial DNA cyt $b$ gene sequences provide evidence for genetic differentiation of three Vimba species, as in $V$. vimba, V. mirabilis, and V. melanops are determined: 7, 7, and 1 , respectively. The haplotype and nucleotide diversity are $\mathrm{Hd}=0.855 \pm 0.025$ and $\pi=0.00175 \pm 0.00029$ for Vimba vimba, $\mathrm{Hd}=0.939 \pm 0.00333$ and $\pi=0.00783 \pm 0.00119$ for $V$. melanops, and $\mathrm{Hd}=0.154 \pm 0.01590$ and $\pi=0.00015 \pm 0.00012$ for $V$. mirabilis. The overall haplotype and nucleotide diversity are $\mathrm{Hd}=0.907 \pm 0.015$ and $\pi=0.00994 \pm 0.00104$. According to the AMOVA, $88.34 \%$ of all genetic variation occurs among three species. In the haplotype network analysis, the 29 distinct haplotypes are separated by one up to nine mutations. The most common haplotype was $\mathrm{H} 1$, shared by many populations belonging to $V$. vimba. Many haplotypes were distributed in either one or two populations (Fig. 2). Average pairwise genetic distances between species were estimated as $0.0212(\mathrm{~V}$. vimba and $V$. melanops), 0.0198 ( $V$. vimba and $V$. mirabilis), and 0.0171 ( $V$. melanops and $V$. mirabilis). Average pairwise genetic distance was estimated as 0.0101 between $V$. vimba Caspian (H16) and West lineages. Average intraspecific variations within $V$. vimba, $V$. mirabilis, and $V$. melanops were estimated as $0.002,0.000$, and 0.008 , respectively. Nucleotide sequences of the COI barcoding region $(652 \mathrm{bp})$ were obtained in 67 specimens from three species of Vimba (Additional Table 1). The nucleotide frequencies were calculated as $25.28 \% \mathrm{~A}, 29.00 \% \mathrm{~T}$, $27.71 \% \mathrm{C}$, and $18.01 \% \mathrm{G}$. The transition/transversion rate $\mathrm{k} 1=3.588$ (purines), $\mathrm{k} 2=1.848$ (pyrimidines), and the overall transition/transversion bias was calculated as $\mathrm{R}=$ 1.27. The haplotype number was identified as 13: six from $V$. vimba $(\mathrm{N}=46)$, five from $V$. melanops $(\mathrm{N}=16)$, and two from $V$. mirabilis $(\mathrm{N}=5)$ (Table 1$)$. A total of 21 variable sites were recognized, and 10 of them were parsimony-informative. The haplotype and nucleotide diversity with the standard deviation are $\mathrm{Hd}=0.680 \pm 0.00156$ and $\pi=0.00308 \pm 0.00021$ for $V$. vimba, $\mathrm{Hd}=0.608 \pm 0.01695$ and $\pi=0.00204 \pm 0.00065$ for $V$. melanops, and $\mathrm{Hd}=0.400$ \pm 0.237 and $\pi=0.00123 \pm 0.00073$ for $V$. mirabilis. The overall haplotype and nucleotide diversity are $\mathrm{Hd}$ : 0.826 \pm 0.026 and $\pi=0.00457 \pm 0.00041$. According to the AMOVA, $58.52 \%$ of all observed genetic variations in three species are occurring within species. Haplotype network analysis has 13 unique haplotypes with at least one mutational step, and no haplotype is shared between species that are separated from each other by at least one mutational step. The most common haplotypes were H13, represented by 20 specimens sampled by İznik Lake. All haplotypes were distributed in either one or two populations (Fig. 3). Average pairwise genetic distances between species were estimated as 0.0054 ( $V$. vimba and $V$. melanops), 0.0093 ( $V$. vimba and $V$. mirabilis), and 0.0088 ( $V$. melanops and $V$. mirabilis).

\section{Phylogenetic relationships and divergence times}

Based on ML and Bayesian phylogenetic inferences, four mitochondrial clades of Vimba species are observed. The first and second clades include distinct $V$. vimba 


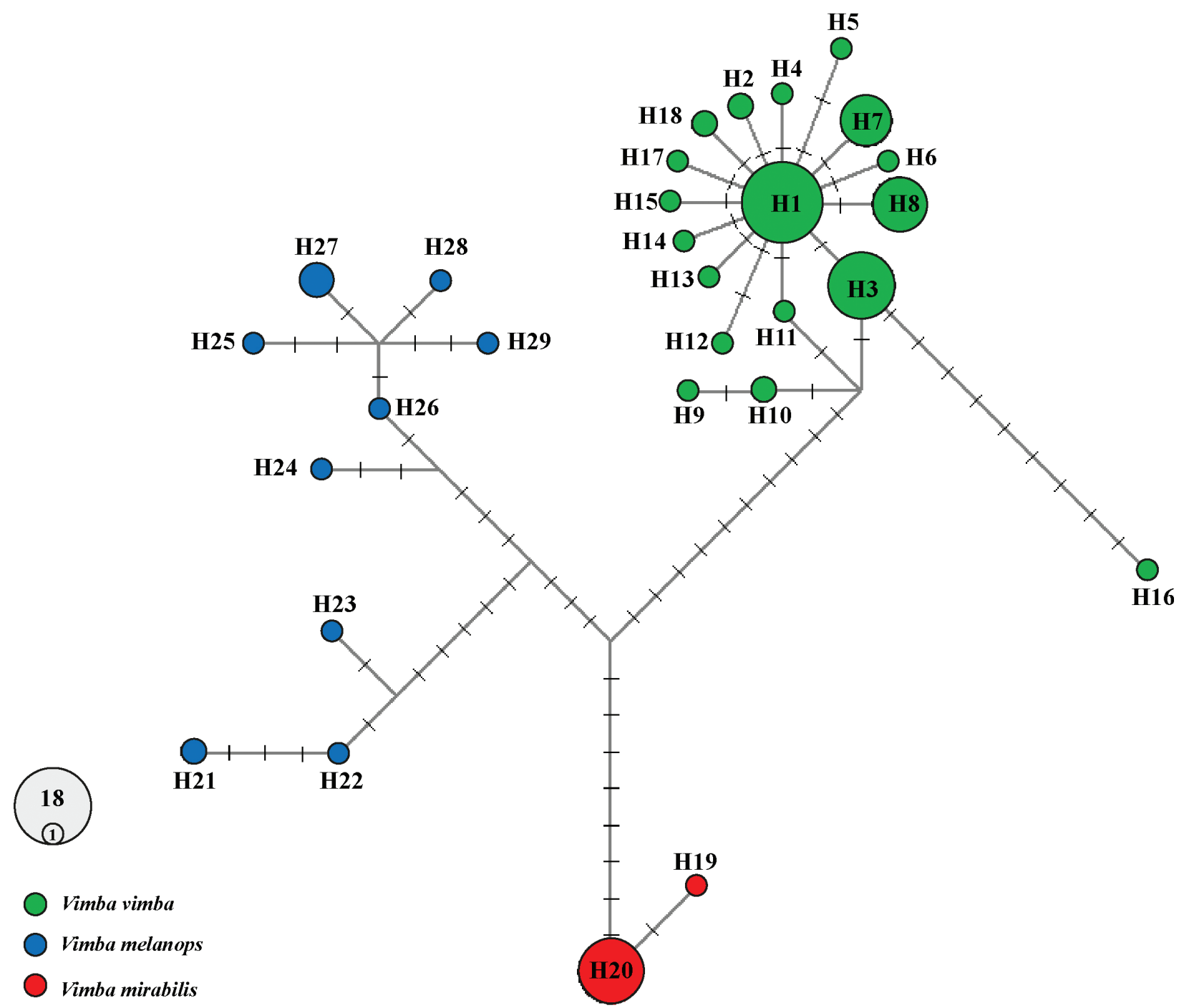

Figure 2. Median-joining network of the cyt b haplotypes. Circle size corresponds to sample size; one bar indicates an additional mutational step. Each small line represents one nucleotide difference.

populations, while the other clades comprise $V$. mirabilis and $V$. melanops. $V$. vimba is not monophyletic because the Western (Pontic) and Caspian basin haplotypes of $V$. vimba are located in two different clades. As shown in Fig. 4, the maximum likelihood and Bayesian inference analyses of concatenated data of mitochondrial cyt $b$ and COI barcoding region trees were supported by high bootstrap values $(>75)$ for the distinction of species and Vimba vimba lineages. According to the divergence time between the two main clades, the divergence among the Vimba species starts in 2.27 (0.57-4.02) mya, early Pleistocene (Gelasian), and differentiates into four clades in early Pleistocene (Calabrian) based on StarBEAST analysis (Fig. 5).

\section{Discussion}

The present study investigates the phylogeny and phylogeography of three Vimba species to provide the first comprehensive molecular study on the genus. Although some work has been conducted on Vimba species, there are no comprehensive studies on their phylogeography, which is provided in the present study.

Here, the phylogeny constructed by ML and BI approaches is based on concatenated mtDNA cyt $b$ and COI sequences of three Vimba species. The phylogenetic tree topology revealed that species were clustered into four well-supported clades ( $V$. vimba (Western), $V$. vimba (Caspian), V. melanops, and V. mirabilis) with high-reliability BI Posterior probability value $(\mathrm{BI}=0.98)$ although low ML bootstrap value $(\mathrm{ML}<50)$. In $V$. vimba, two lineages are observed, the Caspian and the Western, monophyly of both group supported by high BI (0.98) value. $V$. melanops and $V$. mirabilis belong to different clades with high BP (97) and BI posterior probability (1.00) (Fig. 4). According to tree topology, a clear geographical pattern emerged, which is also supported by the median joining network. However, variability at cyt $\mathrm{b}$ and COI markers varied, the AMOVA analysis found almost all $(88.34 \%)$ of the genetic variations observed for the cyt $b$ gene occurred between species, while this value drops to $58.52 \%$ for the COI barcoding region. 


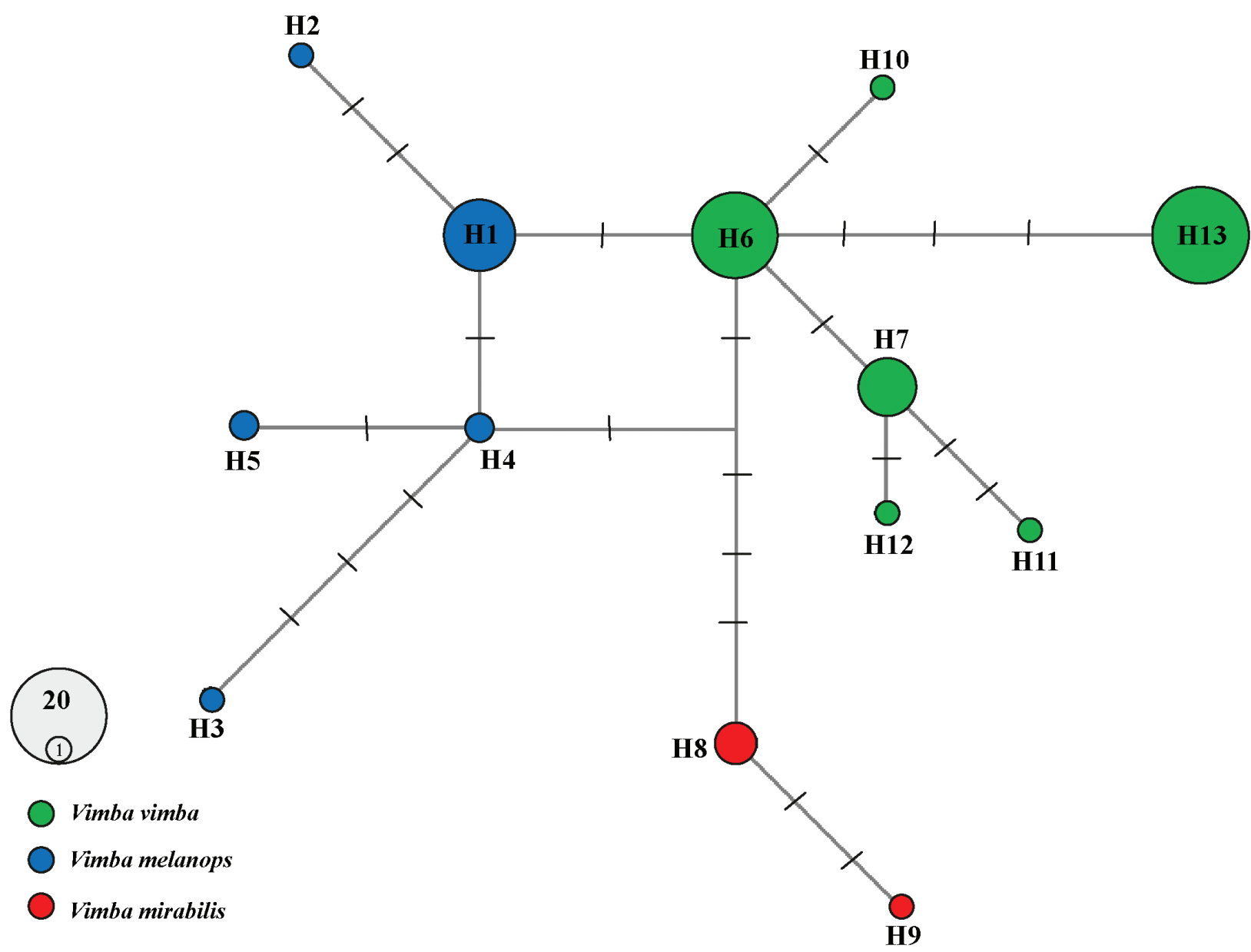

Figure 3. Median-joining network of the COI haplotypes. Circle size corresponds to sample size; one bar indicates an additional mutational step. Each small line represents one nucleotide difference.

Geiger et al. (2014) similarly reported that $V$. vimba, $V$. melanops, $V$. mirabilis are closely related species, with relatively low interspecific genetic distances, but they can be distinguished from each other by morphological characters. Species with high haplotype diversity and low nucleotide diversity are usually thought to originate through small founder populations during the expansion period, in accordance with previous analyses of Anatolian freshwater fish species (Hrbek et al. 2004; Bektas et al. 2017). A small independent evolution of similar origin (originating from the Pliocene/Pleistocene) may account for the low intraspecific diversity and corresponds with tectonic or glacial activity in the area. Anatolia and Central Europe were connected from the late Oligocene to the late Miocene (23-5.33 mya) (Popov et al. 2004). The estimated separation time of Vimba from sister genus Blicca was calculated as $6.16(1.42-13.11)$ mya, a timeframe consistent with a dispersal from the Danube basin to Anatolia via river capture during this period (Levy et al. 2009). Similarly, Hänfling et al. (2009) estimated the time of separation of Vimba genus in Pleistocene as well. The divergence time between the two main clades shows that the divergence among the Vimba species started in 2.27 (0.57-4.02) mya, early Pleistocene (Gelasian) (Fig. 5).
According to Hänfling et al. (2009), V. vimba lineages were defined as Pontic and also Caspian clade, including Caspian Sea haplotype which is highly divergent from the other haplogroup. This suggests $V$. vimba originated from two refugial regions located in the Danubian drainage and the northern Pontic regions. Moreover, Hänfling et al. (2009), Naseka and Bogutskaya (2009), Jouladeh-Roudbar et al. (2015), and Esmaeili et al. (2018) stated that the Caspian Vimba should be considered as a distinct species corresponding to $V$. persa, which is anadromous and endemic to the Caspian Sea. Similarly, in this study, it is supported that Caspian Vimba should be classified as $V$. persa, not $V$. vimba considering intra and interspecies divergence of Vimba species. Furthermore, both phylogenetic and StarBEAST analyses support Caspian Vimba corresponding to a different linage from the Western lineage of $V$. vimba. However, advanced research and comprehensive sampling of Caspian and Western populations are required to clarify this. $V$. vimba in other regions has relatively low intraspecific diversity, except for the Caspian haplogroup. Natural distribution records for $\mathrm{V}$. vimba in Europe, the former USSR, are most likely due to stock translocations and introductions from other areas due to their economic value (Freyhof 1999). 


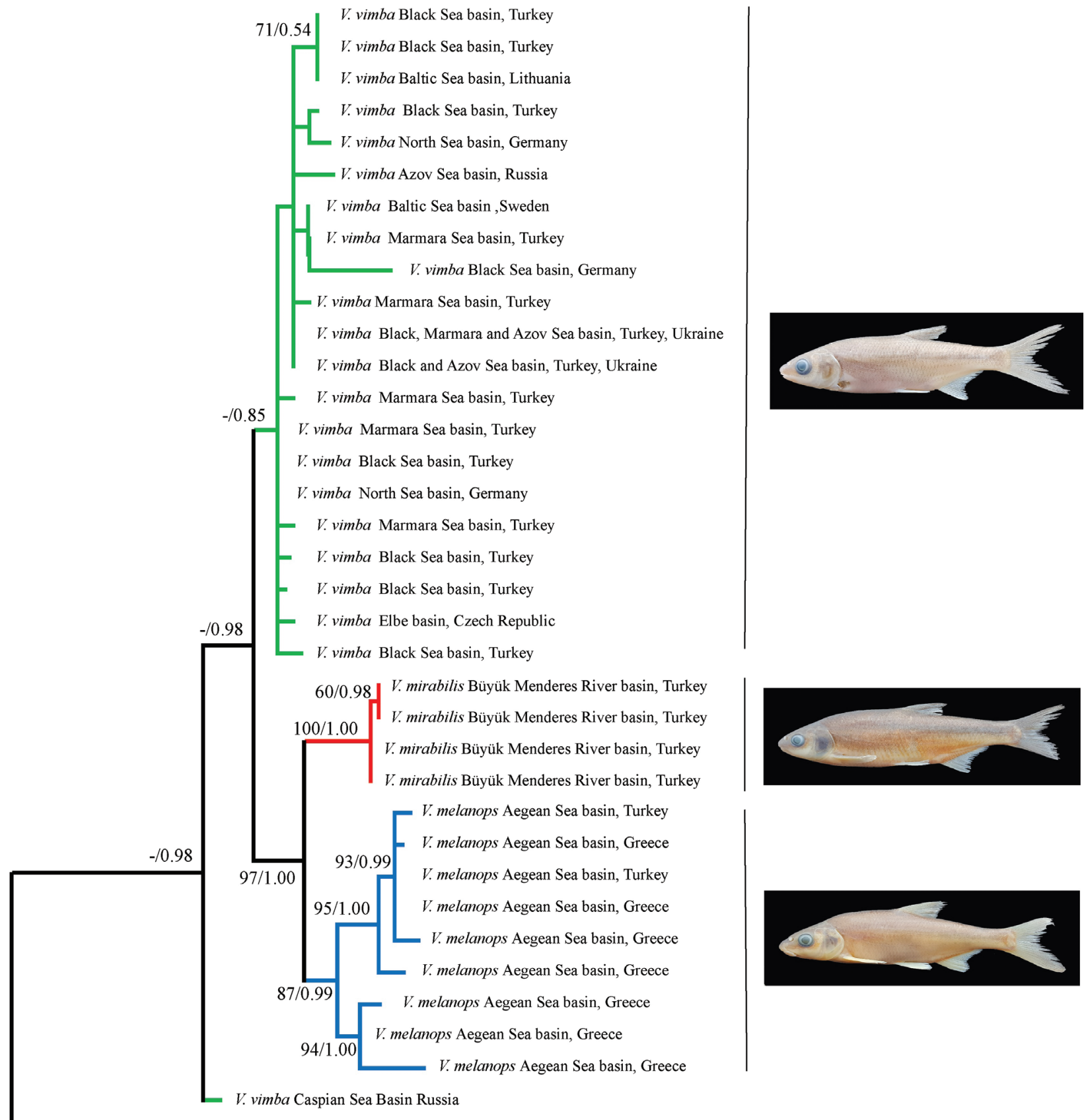

Blicca bjoerkna AP009304

0.010

Figure 4. Maximum likelihood tree based on the two concatenated mitochondrial genes (cyt b and COI) (1675 bp) sequences of Vimba species. Maximum likelihood and Bayesian inference analyses resulted in congruent trees. Bootstrap and posterior probability values are shown above nodes on a tree if $50 \%$ or higher.

Phylogeography of $V$. vimba was investigated by Hänfling et al. (2009), and it reflected the presence of two refugia and recolonization of $V$. vimba as Caspian sea, and Western or Pontid clade, dating back 1-2 mya during the early Pleistocene. According to the present study, the separation of Vimba, dated in Pleistocene, occurred at 1.06 (0.2-2.55) mya. Correspondingly, in view of the timing, Pleistocene events must have played a central role in structuring the Balkan's marbled goby populations (Vanhove et al. 2012). In addition, multiples fish groups in the Black and Caspian Sea basins display similar patterns of Pleistocene divergence such as the Black Sea roach, Rutilus frisii, salmon, barbell, and dreissenid mussels (Stepien et al. 2003; Kotlík et al. 2008; Ninua et al. 2018; Bartáková et al. 2019; Levin et al. 2019). Due to the migration of Vimba using brackish waters, it spread all over Eurasia also in Northern Germany and Netherlands, using the Danube River and old canal system (Freyhof 1999).

In the Early Pleistocene, the depression of the Marmara Sea and the uplift of the Aegean mountains contributed 


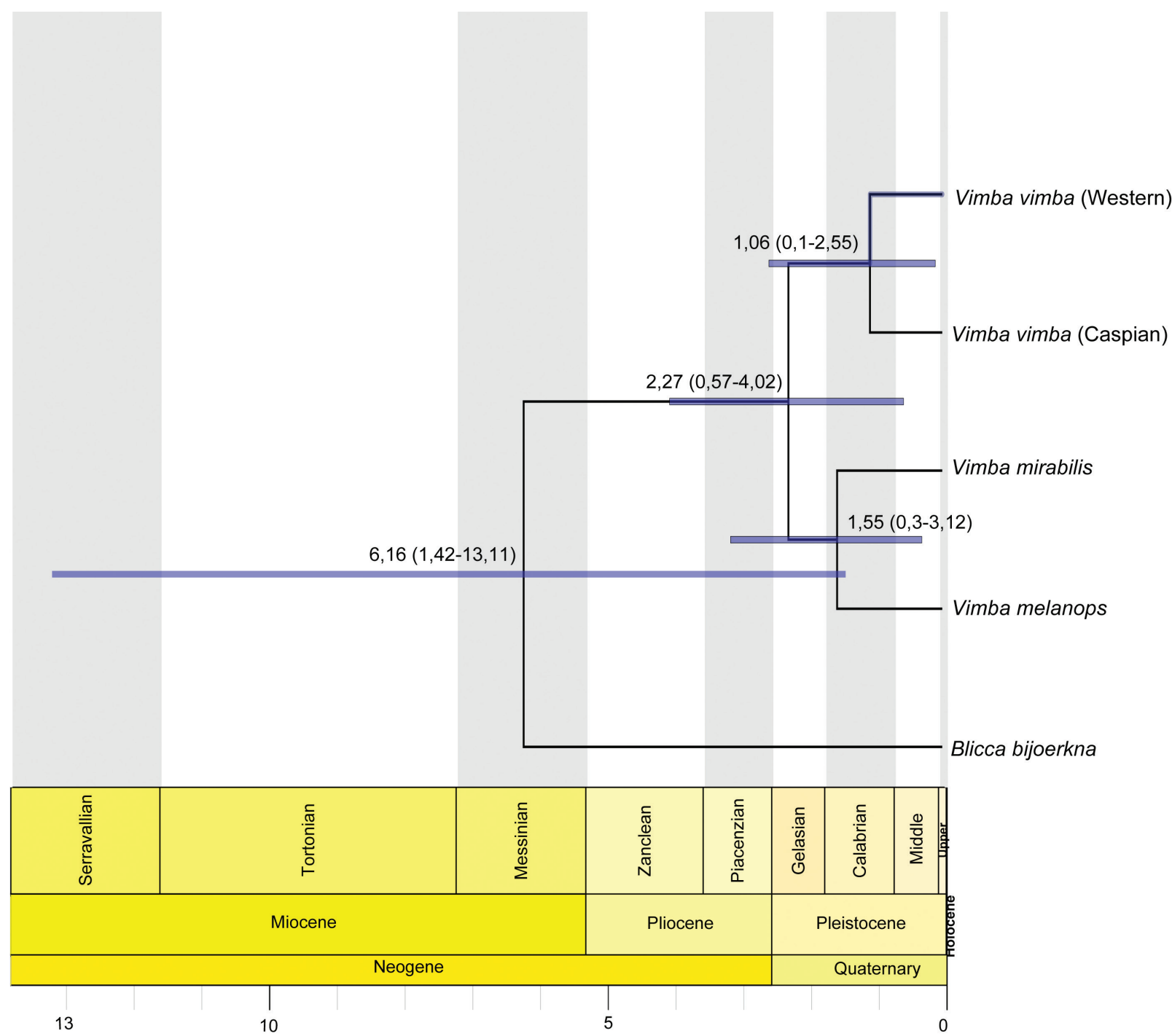

Figure 5. Divergence timescale for the Vimba species inferred under Bayesian strict clock method from two concatenated mitochondrial genes (cyt b and COI) (1675 bp) sequences. Numbers in front of the node represent divergence times in million years (Ma) and their HPD 95\% credibility intervals.

greatly to the separation of $V$. vimba and $V$. mirabilis. Furthermore, while the water of the Büyük Menderes River was flowing in the north-south direction because of the western Anatolian Mountains barrier, fractures and foldings which occurred in the Early Pleistocene caused the river to turn west and take its present form. $V$. mirabilis is present in Bafa Lake because of alluvium brought in by the Menderes River that blocked the old sea gulf and separated it from the sea, creating Bafa Lake (Akçer-Ön et al. 2020).

Por, (1989) specified that a line drawn from west to east in the middle of Turkey could be considered as a major suture, which leads to distinct species of Vimba between the Büyük Menderes and northern Aegean. The differences between the Balkan and Anatolian species can primarily be associated with the formation of the Aegean in the late Pliocene (Kosswig 1955; Bilgin 2011). Also, Bektas et al. (2019) discovered that dispersal of Alburnoides symrnae and Alburnoides economui was dated in 5.42-2.31 Ma (Early Pliocene), when the former Aegeopotamus River was a very large river that discharged the waters of $\mathrm{Pa}$ ratethys into the Aegean Sea. Divergence time estimated between $V$. melanops and $V$. mirabilis as 1.69 (1.07-2.38) mya is consistent with this event. Durand et al. (2000), Hewitt (2000), and Hewitt (2004) further highlighted that the Danube was an essential component of the Chub (Squalius cephalus) evolutionary history, especially during the Pleistocene glaciations as it acted as a refugium for this species. Our molecular data confirm this influence on the divergence of $V$. melanops and $V$. mirabilis.

\section{Acknowledgements}

I am pleased to thank Davut Turan and Cüneyt Kaya for providing samples. Also, I extend my special thanks to the Editor, Nicolas Hubert and two anonymous reviewers for their valuable comments and suggestions that greatly improved the manuscript. 


\section{References}

Akçer-Ön S, Greaves AM, Manning SW, Ön ZB, Çağatay MN, Sakınç M, Oflaz A, Tunoğlu C, Salihoğlu R (2020) Redating the formation of Lake Bafa, western Turkey: Integrative geoarchaeological methods and new environmental and dating evidence. Geoarchaeology 35: 659-677. https://doi.org/10.1002/gea.21791

Aksu I, Bektaş Y (2019) Mitochondrial phylogeny and biogeography of the genus Gobio (Teleostei: Cyprinidae) in Turkey. Zoology in the Middle East 65: 128-141. https://doi.org/10.1080/09397140.20 19.1586126

Bandelt HJ, Forster P, Röhl A (1999) Median-joining networks for inferring intraspecific phylogenies. Molecular Biology and Evolution 16: 37-48. https://doi.org/10.1093/oxfordjournals.molbev.a026036

Bartáková V, Bryja J, Šanda R, Bektas Y, Stefanov T, Choleva L, Smith C, Reichard M (2019) High cryptic diversity of bitterling fish in the southern West Palearctic. Molecular Phylogenetics and Evolution 133: 1-11. https://doi.org/10.1016/j.ympev.2018.12.025

Bektas Y, Turan D, Aksu I, Ciftci Y, Eroglu O, Kalayci G, Belduz AO (2017) Molecular phylogeny of the genus Capoeta (Teleostei: Cyprinidae) in Anatolia, Turkey. Biochemical Systematics and Ecology 70: 80-94. https://doi.org/10.1016/j.bse.2016.11.005

Bektas Y, Aksu I, Kaya C, Baycelebi E, Atasaral S, Ekmekci FG, Turan D (2019) Phylogeny and phylogeography of the genus Alburnoides (Teleostei, Cyprinidae) in Turkey based on mitochondrial DNA sequences. Mitochondrial DNA Part A: DNA Mapping, Sequencing, and Analysis 30: 794-805. https://doi.org/10.1080/24701394.2019 .1664493

Bell MA, Lloyd GT (2015) Strap: An R package for plotting phylogenies against stratigraphy and assessing their stratigraphic congruence. Palaeontology 58: 379-389. https://doi.org/10.1111/pala.12142

Bilgin R (2011) Back to the suture: The distribution of intraspecific genetic diversity in and around Anatolia. International Journal of Molecular Sciences 12: 4080-4103. https://doi.org/10.3390/ ijms 12064080

Bogutskaya NG (1997) Contribution to the knowledge of leuciscine fishes of Asia Minor. Part 2. An annotated check-list of leuciscine fishes (Leuciscinae, Cyprinidae) of Turkey with descriptions of a new species and two new subspecies. Mitteilungen aus dem Hamburgischen Zoologischen Museum und Institut 94: 161-186.

Bouckaert R, Heled J, Kühnert D, Vaughan T, Wu CH, Xie D, Suchard MA, Rambaut A, Drummond AJ (2014) BEAST 2: A Software Platform for Bayesian Evolutionary Analysis. PLoS Computational Biology 10: e1003537. https://doi.org/10.1371/journal. pcbi. 1003537

Buj I, Šanda R, Zogaris S, Freyhof J, Geiger MF, Vukić J (2019) Cryptic diversity in Telestes pleurobipunctatus (Actinopterygii; Leuciscidae) as a consequence of historical biogeography in the Ionian Freshwater Ecoregion (Greece, Albania). Hydrobiologia 835: 147163. https://doi.org/10.1007/s10750-019-3935-6

Chaichi AR, Vosoughi GKK, Jamili S, Fazli H (2011a) Population dynamics of Vimba vimba persa in Iranian waters of the Caspian Sea. Cybium: International Journal of Ichthyology 35: 237-243.

Chaichi AR, Vosoughi GH, Kaymaram F, Jamili S, Fazli H (2011b) Reproduction characteristics of the Vimba vimba persa (Pallas, 1811), in coastal waters of the Caspian Sea. Iranian Journal of Fisheries Sciences 10: 585-595.
Durand JD, Tsigenopoulos CS, Ünlü E, Berrebi P (2002) Phylogeny and biogeography of the family cyprinidae in the Middle East inferred from cytochrome b DNA - Evolutionary significance of this region. Molecular Phylogenetics and Evolution 22: 91-100. https:// doi.org/10.1006/mpev.2001.1040

Durand JD, Unlu E, Doadrio I, Pipoyan S, Templeton AR (2000) Origin, radiation, dispersion and allopatric hybridization in the chub Leuciscus cephalus. Proceedings of the Royal Society B: Biological Sciences 267: 1687-1697. https://doi.org/10.1098/rspb.2000.1196

Esmaeili HR, Sayyadzadeh G, Eagderi S, Abbasi K (2018) Checklist of freshwater fishes of Iran. FishTaxa 3: 1-95.

Excoffier L, Lischer HEL (2010) Arlequin suite ver 3.5: A new series of programs to perform population genetics analyses under Linux and Windows. Molecular Ecology Resources 10: 564-567. https://doi. org/10.1111/j.1755-0998.2010.02847.x

Freyhof J (1999) Records of Vimba vimba (Teleostei, Cyprinidae) in the River Rhine and its tributaries. Folia Zoologica 48: 315-320.

Geiger MF, Herder F, Monaghan MT, Almada V, Barbieri R, Bariche M, Berrebi P, Bohlen J, Casal-Lopez M, Delmastro GB, Denys GPJJ, Dettai A, Doadrio I, Kalogianni E, Kärst H, Kottelat M, Kovačić M, Laporte M, Lorenzoni M, Marčić Z, Özuluğ M, Perdices A, Perea S, Persat H, Porcelotti S, Puzzi C, Robalo J, Šanda R, Schneider M, Šlechtová V, Stoumboudi M, Walter S, Freyhof J (2014) Spatial heterogeneity in the mediterranean biodiversity hotspot affects barcoding accuracy of its freshwater fishes. Molecular Ecology Resources 14: 1210-1221. https://doi.org/10.1111/1755-0998.12257

Guindon S, Dufayard JF, Lefort V, Anisimova M, Hordijk W, Gascuel O (2010) New algorithms and methods to estimate maximum-likelihood phylogenies: Assessing the performance of PhyML 3.0. Systematic Biology 59: 307-321. https://doi.org/10.1093/sysbio/syq010

Hänfling B, Dümpelmann C, Bogutskaya NG, Brandl R, Brändle M (2009) Shallow phylogeographic structuring of Vimba vimba across Europe suggests two distinct refugia during the last glaciation. Journal of Fish Biology 75: 2269-2286. https://doi.org/10.1111/j.10958649.2009.02415.x

Hasegawa M, Kishino H, Yano T-aki (1985) Dating of the human-ape splitting by a molecular clock of mitochondrial DNA. Journal of Molecular Evolution 22: 160-174. https://doi.org/10.1007/ BF02101694

Hewitt G (2000) The genetic legacy of the quaternary ice ages. Nature 405: 907-913. https://doi.org/10.1038/35016000

Hewitt GM (2004) Genetic consequences of climatic oscillations in the Quaternary. Philosophical Transactions of the Royal Society B: Biological Sciences 359: 183-195. https://doi.org/10.1098/ rstb.2003.1388

Jouladeh-Roudbar A, Vatandoust S, Eagderi S, Jafari-Kenari S, Mousavi-Sabet H (2015) Freshwater fishes of Iran; an updated checklist. AACL Bioflux 8: 855-909.

Kosswig C (1955) Zoogeography of the near east. Systematic Zoology 4: 49-73. https://doi.org/10.2307/sysbio/4.2.49

Kotlík P, Marková S, Choleva L, Bogutskaya NG, Ekmekçi FG, Ivanova PP (2008) Divergence with gene flow between Ponto-Caspian refugia in an anadromous cyprinid Rutilus frisii revealed by multiple gene phylogeography. Molecular Ecology 17: 1076-1088. https:// doi.org/10.1111/j.1365-294X.2007.03638.x

Kumar S, Stecher G, Li M, Knyaz C, Tamura K (2018) MEGA X: Molecular evolutionary genetics analysis across computing platforms. 
Molecular Biology and Evolution 35: 1547-1549. https://doi. org $/ 10.1093 / \mathrm{molbev} / \mathrm{msy} 096$

Levin BA, Freyhof J, Lajbner Z, Perea S, Abdoli A, Gaffaroĝlu M, Özuluĝ M, Rubenyan HR, Salnikov VB, Doadrio I (2012) Phylogenetic relationships of the algae scraping cyprinid genus Capoeta (Teleostei: Cyprinidae). Molecular Phylogenetics and Evolution 62: 542-549. https://doi.org/10.1016/j.ympev.2011.09.004

Levin BA, Gandlin AA, Simonov ES, Levina MA, Barmintseva AE, Japoshvili B, Mugue NS, Mumladze L, Mustafayev NJ, Pashkov AN, Roubenyan HR, Shapovalov MI, Doadrio I (2019) Phylogeny, phylogeography and hybridization of Caucasian barbels of the genus Barbus (Actinopterygii, Cyprinidae). Molecular Phylogenetics and Evolution 135: 31-44. https://doi.org/10.1016/j.ympev.2019.02.025

Levy A, Doadrio I, Almada VC (2009) Historical biogeography of European leuciscins (Cyprinidae): Evaluating the Lago Mare dispersal hypothesis. Journal of Biogeography 36: 55-65. https://doi. org/10.1111/j.1365-2699.2008.01969.x

Mohamadian S, Rezavani Gilkolaei S, Rouhollahi S, Taghavi MJ, Nayerani M, Shirzad E, Taheri Mirghaed A (2012) Genetic characterization of Vimba vimba persa (Pallas, 1814) in Southern parts of the Caspian Sea using microsatellite markers. Iranian Journal of Fisheries Sciences 11: 347-357.

Naseka A, Bogutskaya N (2009) Fishes of the Caspian Sea: zoogeography and updated check-list. Zoosystematica Rossica 18: 295-317. https://doi.org/10.31610/zsr/2009.18.2.295

Ninua L, Tarkhnishvili D, Gvazava E (2018) Phylogeography and taxonomic status of trout and salmon from the Ponto-Caspian drainages, with inferences on European Brown Trout evolution and taxonomy. Ecology and Evolution 8: 2645-2658. https://doi.org/10.1002/ ece 3.3884

Ogilvie HA, Bouckaert RR, Drummond AJ (2017) StarBEAST2 brings faster species tree inference and accurate estimates of substitution rates. Molecular Biology and Evolution 34: 2101-2114. https://doi. org/10.1093/molbev/msx126

Perea S, Böhme M, Zupančič P, Freyhof J, Šanda R, Özulu M, Abdoli A, Doadrio I (2010) Phylogenetic relationships and biogeographical patterns in Circum-Mediterranean subfamily Leuciscinae (Teleostei, Cyprinidae) inferred from both mitochondrial and nuclear data. BMC Evolutionary Biology 10: e265. https://doi.org/10.1186/14712148-10-265

Por DFF (1989) The legacy of Tethys. 41-64 pp. https://doi. org/10.1007/978-94-009-0937-3

Rambaut A (2018) FigTree 1.4.4 software. Institute of Evolutionary Biology, Univ. Edinburgh.

Rambaut A, Drummond AJ (2013) Tracer v1.6. [Available from] http:// tree.bio.ed.ac.uk/software/tracer/
Ronquist F, Huelsenbeck JP (2003) MrBayes 3: Bayesian phylogenetic inference under mixed models. Bioinformatics 19: 1572-1574. https://doi.org/10.1093/bioinformatics/btg180

Rozas J, Ferrer-Mata A, Sanchez-DelBarrio JC, Guirao-Rico S, Librado P, Ramos-Onsins SE, Sanchez-Gracia A (2017) DnaSP 6: DNA sequence polymorphism analysis of large data sets. Molecular Biology and Evolution 34: 3299-3302. https://doi.org/10.1093/molbev/msx248

Schönhuth S, Vukić J, Šanda R, Yang L, Mayden RL (2018) Phylogenetic relationships and classification of the Holarctic family Leuciscidae (Cypriniformes: Cyprinoidei). Molecular Phylogenetics and Evolution 127: 781-799. https://doi.org/10.1016/j.ympev.2018.06.026

Stepien C, Taylor CD, Grigorovich I, Shirman SV, Wei R, Korniushin A V, Dabrowska KA (2003) DNA and systematic analysis of invasive and native dreissenid mussels: Is Dreissena bugensis really D. rostriformis? Aquatic Invaders 14: 8-18.

Tamura K, Nei M (1993) Estimation of the number of nucleotide substitutions in the control region of mitochondrial DNA in humans and chimpanzees. Molecular Biology and Evolution 10: 512-526. https://doi.org/10.1093/oxfordjournals.molbev.a040023

Triantafyllidis A, Bobori D, Koliamitra C, Gbandi E, Mpanti M, Petriki O, Karaiskou N (2011) DNA barcoding analysis of fish species diversity in four north Greek lakes. Mitochondrial DNA 22: 37-42. https://doi.org/10.3109/19401736.2010.542242

Tsigenopoulos CS, Kotlík P, Berrebi P (2002) Biogeography and pattern of gene flow among Barbus species (Teleostei: Cyprinidae) inhabiting the Italian Peninsula and neighbouring Adriatic drainages as revealed by allozyme and mitochondrial sequence data. Biological Journal of the Linnean Society 75: 83-99. https://doi.org/10.1046/ j.1095-8312.2002.00007.x

Turan D, Kalayci G, Kaya C, Bektaş Y, Küçük F (2018) A new species of Petroleuciscus (Teleostei: Cyprinidae) from the Büyük Menderes River, southwestern Anatolia, Turkey. Journal of Fish Biology 92: 875-887. https://doi.org/10.1111/jfb.13525

Vanhove MPM, Economou AN, Zogaris S, Larmuseau MHD, Giakoumi S, Kalogianni E, Volckaert FAM, Huyse T (2012) Phylogenetics and biogeography of the Balkan 'sand gobies'(Teleostei: Gobiidae): vulnerable species in need of taxonomic revision. Biological Journal of the Linnean Society 105: 73-91. https://doi.org/10.1111/j.1095-8312.2011.01781.x

Viñuela Rodríguez N, Šanda R, Zogaris S, Vukić J (2020) Distribution and genetic diversity of two species of Pelasgus minnows (Leuciscidae) in southern Greece. Knowledge and Management of Aquatic Ecosystems 2020-Janua. https://doi.org/10.1051/kmae/2020019

Zardoya R, Doadrio I (1999) Molecular evidence on the evolutionary and biogeographical patterns of European cyprinids. Journal of Molecular Evolution 49: 227-237. https://doi.org/10.1007/ PL00006545 